\title{
Numerical Solution of Pantograph-Type Delay Differential Equations Using Perturbation-Iteration Algorithms
}

\author{
M. Mustafa Bahşi ${ }^{1}$ and Mehmet Çevik ${ }^{2}$ \\ ${ }^{1}$ Department of Mechanical Engineering, Celal Bayar University, 45140 Manisa, Turkey \\ ${ }^{2}$ Department of Mechanical Engineering, Izmir Katip Çelebi University, 35620 Izmir, Turkey \\ Correspondence should be addressed to Mehmet Çevik; cevik2002@gmail.com
}

Received 12 August 2015; Accepted 16 November 2015

Academic Editor: Vasile Marinca

Copyright ( 2015 M. M. Bahşi and M. Çevik. This is an open access article distributed under the Creative Commons Attribution License, which permits unrestricted use, distribution, and reproduction in any medium, provided the original work is properly cited.

The pantograph equation is a special type of functional differential equations with proportional delay. The present study introduces a compound technique incorporating the perturbation method with an iteration algorithm to solve numerically the delay differential equations of pantograph type. We put forward two types of algorithms, depending upon the order of derivatives in the Taylor series expansion. The crucial convenience of this method when compared with other perturbation methods is that this method does not require a small perturbation parameter. Furthermore, a relatively fast convergence of the iterations to the exact solutions and more accurate results can be achieved. Several illustrative examples are given to demonstrate the efficiency and reliability of the technique, even for nonlinear cases.

\section{Introduction and Preliminaries}

The pantograph equation is a special type of functional differential equations with proportional delay. It arises in rather different fields of pure and applied mathematics, such as electrodynamics, control systems, number theory, probability, and quantum mechanics. Many researchers have studied the pantograph-type delay differential equation using analytical and numerical techniques [1-8]. The second-order pantograph-type delay differential equation is given as $[7,8]$

$$
\frac{d^{2} u(x)}{d x^{2}}=f(x, u(x), u(\alpha x)), \quad x \in(0, T)
$$

with the boundary conditions

$$
\begin{aligned}
& u(0)=b, \\
& u(T)=c,
\end{aligned}
$$

where $T>0$ and $0<\alpha<1$.
A brief review of recent literature on the methods of solution for pantograph-type delay differential equations can be found in the paper by Trif [9].

On the other hand, perturbation methods [10] have been among the most common approximate methods used for solving algebraic equations, differential equations, integrodifferential equations, and difference equations. The primary limitation of these methods is the necessity of a small parameter. This parameter might come out as an original physical parameter of the given equation or, alternatively, it may be inserted as an artificial parameter. Several different methods have been established in order to deal with this limitation.

In this study, we present the application of a hybrid technique combining the perturbation method with an iteration algorithm to find a numerical solution for pantograph-type delay differential equations. Various researchers considered this new perturbation-iteration method [11-16]. Pakdemirli and coworkers, besides others, have studied extensively this method. The first study on algebraic equations was carried out 
by Pakdemirli and Boyac1 [17] where they proposed new root finding algorithms using the new systematic approach. Later, fourth-order [18] and fifth-order [19] derivative algorithms were presented. Aksoy and Pakdemirli [20] systematically generated the present method for both linear and nonlinear second-order differential equations and applied to it Bratutype equations. Dolapçi et al. [21] used this method to solve Fredholm and Volterra integral equations and argued that it can be applied to both types of integral equations.

The main purpose of the work presented in this paper is to apply the new perturbation-iteration solution for the pantograph-type delay differential equations. To the authors' knowledge, the application of perturbation-iteration algorithms to pantograph-type delay differential equations is novel. The two types of perturbation-iteration algorithms, $\operatorname{PIA}(1,1)$ and $\operatorname{PIA}(1,2)$, are introduced in the next section. Then, the method is applied to pantograph equations via six examples, two of which are first-order linear, one is first-order nonlinear, and the others are second-order nonlinear; they showed excellent agreement with the published results and verified the accuracy and efficiency of the present method. The results are discussed and commented on.

The general form of pantograph-type delay differential equations of both first- and second-order, including (1), can be stated as

$$
F\left(u^{\prime \prime}, u^{\prime}, u, u_{\alpha}, \varepsilon, x\right)=0
$$

where $u=u(x), u_{\alpha}=u(\alpha x), 0<\alpha<1$ is a given constant, and $\varepsilon$ is the perturbation parameter. In this study, we investigated a solution for (3) which is closed form. Equation (3) covers many different problems studied by various researchers.

\section{Perturbation-Iteration Algorithms}

Perturbation-iteration algorithms are briefly called $\operatorname{PIA}(n, m)$. Here, $n$ denotes the number of correction terms in the perturbation expansion and $m$ denotes the order of derivatives in the Taylor series expansion. Generally, $n$ is smaller than or equal to $m$; otherwise, the correction terms cannot be calculated. In this study, $\operatorname{PIA}(1,1)$ and $\operatorname{PIA}(1,2)$ algorithms constructed by taking correction term in the perturbation expansion together with first- and second-order derivatives, respectively, are employed.

2.1. Perturbation-Iteration Algorithm $\operatorname{PIA}(1,1)$. In $\operatorname{PIA}(1,1)$ algorithm, we propose a perturbation-iteration algorithm by taking one correction term in the perturbation expansion and correction terms of only the first derivatives in the Taylor expansion; that is, $n=1, m=1$. Let us consider a secondorder pantograph differential equation written in the form of (3). Taking only one correction term in the perturbation expansion, the straightforward expansion for the solution of each iteration can be written as follows:

$$
u_{n+1}=u_{n}+\varepsilon\left(u_{c}\right)_{n}
$$

where $\left(u_{c}\right)_{n}$ is the $n$th correction term of the perturbationiteration algorithm. Then, we substitute (4) into (3) and expand it in a Taylor series with first derivatives to obtain

$$
\begin{aligned}
& F\left(u_{n}^{\prime \prime}, u_{n}^{\prime}, u_{n},\left(u_{\alpha}\right)_{n}, 0\right) \\
& +F_{u^{\prime \prime}}\left(u_{n}^{\prime \prime}, u_{n}^{\prime}, u_{n},\left(u_{\alpha}\right)_{n}, 0\right) \varepsilon\left(u_{c}^{\prime \prime}\right)_{n} \\
& +F_{u^{\prime}}\left(u_{n}^{\prime \prime}, u_{n}^{\prime}, u_{n},\left(u_{\alpha}\right)_{n}, 0\right) \varepsilon\left(u_{c}^{\prime}\right)_{n} \\
& +F_{u}\left(u_{n}^{\prime \prime}, u_{n}^{\prime}, u_{n},\left(u_{\alpha}\right)_{n}, 0\right) \varepsilon\left(u_{c}\right)_{n} \\
& +F_{u_{\alpha}}\left(u_{n}^{\prime \prime}, u_{n}^{\prime}, u_{n},\left(u_{\alpha}\right)_{n}, 0\right) \varepsilon\left(\left(u_{\alpha}\right)_{c}\right)_{n} \\
& +F_{\varepsilon}\left(u_{n}^{\prime \prime}, u_{n}^{\prime}, u_{n},\left(u_{\alpha}\right)_{n}, 0\right) \varepsilon=0,
\end{aligned}
$$

where ()$^{\prime}$ denotes differentiation with respect to the independent variable and

$$
\begin{gathered}
F_{u^{\prime \prime}}=\frac{\partial F}{\partial u^{\prime \prime}}, \\
F_{u^{\prime}}=\frac{\partial F}{\partial u^{\prime}}, \\
F_{u}=\frac{\partial F}{\partial u}, \\
F_{u_{\alpha}}=\frac{\partial F}{\partial u_{\alpha}}, \\
F_{\varepsilon}=\frac{\partial F}{\partial \varepsilon} .
\end{gathered}
$$

Bearing in mind that all derivatives are evaluated at $\varepsilon=0$ and rewriting the equation in the following more suitable form

$$
\begin{aligned}
& F_{u^{\prime \prime}}\left(u_{c}^{\prime \prime}\right)_{n}+F_{u^{\prime}}\left(u_{c}^{\prime}\right)_{n}+F_{u}\left(u_{c}\right)_{n}+F_{u_{\alpha}}\left(\left(u_{\alpha}\right)_{c}\right)_{n} \\
& \quad=-F_{\varepsilon}-\frac{F}{\varepsilon},
\end{aligned}
$$

one may easily notice that (7) is a variable coefficient pantograph equation. Starting with an initial guess $u_{0}$, first, we determine $\left(u_{c}\right)_{0}$ from (7) and then substitute it into (4) for calculating $u_{1}$. This iteration procedure is repeated using (4) and (7) until the approximation is sufficient within a userdefined threshold.

2.2. Perturbation-Iteration Algorithm $\operatorname{PIA}(1,2) . \operatorname{In} \operatorname{PIA}(1,2)$ algorithm, we put forward a perturbation-iteration algorithm by taking one correction term in the perturbation expansion and correction terms of up to second derivatives in the Taylor expansion; that is, $n=1, m=2$. Only one correction term perturbation expansion was given before in (4). Now, 
we substitute (4) into (3) and expand in a Taylor series of up to second-order derivatives to obtain

$$
\begin{aligned}
F+ & F_{u^{\prime \prime}} \varepsilon\left(u_{c}^{\prime \prime}\right)_{n}+F_{u^{\prime}} \varepsilon\left(u_{c}^{\prime}\right)_{n}+F_{u} \varepsilon\left(u_{c}\right)_{n} \\
& +F_{u_{\alpha}} \varepsilon\left(\left(u_{\alpha}\right)_{c}\right)_{n}+F_{\varepsilon} \varepsilon+\frac{1}{2} F_{u^{\prime \prime} u^{\prime \prime}} \varepsilon^{2}\left(u_{c}^{\prime \prime}\right)_{n}^{2} \\
& +\frac{1}{2} F_{u^{\prime} u^{\prime}} \varepsilon^{2}\left(u_{c}^{\prime}\right)_{n}^{2}+\frac{1}{2} F_{u u} \varepsilon^{2}\left(u_{c}\right)_{n}^{2} \\
& +\frac{1}{2} F_{u_{\alpha} u_{\alpha}} \varepsilon^{2}\left(\left(u_{\alpha}\right)_{c}\right)_{n}^{2}+\frac{1}{2} \varepsilon^{2} F_{\varepsilon \varepsilon} \\
& +F_{u^{\prime \prime} u^{\prime}} \varepsilon^{2}\left(u_{c}^{\prime \prime}\right)_{n}\left(u_{c}^{\prime}\right)_{n}+F_{u^{\prime \prime} u} \varepsilon^{2}\left(u_{c}^{\prime \prime}\right)_{n}\left(u_{c}\right)_{n} \\
& +F_{u^{\prime \prime} u_{\alpha}} \varepsilon^{2}\left(u_{c}^{\prime \prime}\right)_{n}\left(\left(u_{\alpha}\right)_{c}\right)_{n}+F_{u^{\prime \prime}} \varepsilon^{2}\left(u_{c}^{\prime \prime}\right)_{n} \\
& +F_{u^{\prime} u^{\prime}} \varepsilon^{2}\left(u_{c}^{\prime}\right)_{n}\left(u_{c}\right)_{n}+F_{u^{\prime} u_{\alpha}} \varepsilon^{2}\left(u_{c}^{\prime}\right)_{n}\left(\left(u_{\alpha}\right)_{c}\right)_{n} \\
& +F_{u^{\prime} \varepsilon} \varepsilon^{2}\left(u_{c}^{\prime}\right)_{n}+F_{u u_{\alpha}} \varepsilon^{2}\left(u_{c}\right)_{n}\left(\left(u_{\alpha}\right)_{c}\right)_{n} \\
& +F_{u \varepsilon} \varepsilon^{2}\left(u_{c}\right)_{n}+F_{u_{\alpha} \varepsilon} \varepsilon^{2}\left(\left(u_{\alpha}\right)_{c}\right)_{n}=0 .
\end{aligned}
$$

Then, bearing in mind that all derivatives are evaluated at $\varepsilon=0$, we rewrite this equation in the following more suitable form:

$$
\begin{aligned}
\left(\varepsilon F_{u^{\prime \prime}}\right. & \left.+\varepsilon^{2} F_{u^{\prime \prime} \varepsilon}\right)\left(u_{c}^{\prime \prime}\right)_{n}+\left(\varepsilon F_{u^{\prime}}+\varepsilon^{2} F_{u^{\prime} \varepsilon}\right)\left(u_{c}^{\prime}\right)_{n} \\
& +\left(\varepsilon F_{u}+\varepsilon^{2} F_{u \varepsilon}\right)\left(u_{c}\right)_{n}+\left(\varepsilon F_{u_{\alpha}}+\varepsilon^{2} F_{u_{\alpha} \varepsilon}\right)\left(\left(u_{\alpha}\right)_{c}\right)_{n} \\
& +\frac{1}{2} \varepsilon^{2} F_{u^{\prime \prime} u^{\prime \prime}}\left(u_{c}^{\prime \prime}\right)_{n}^{2}+\frac{1}{2} \varepsilon^{2} F_{u^{\prime} u^{\prime}}\left(u_{c}^{\prime}\right)_{n}^{2} \\
& +\frac{1}{2} \varepsilon^{2} F_{u u}\left(u_{c}\right)_{n}^{2}+\frac{1}{2} \varepsilon^{2} F_{u_{\alpha} u_{\alpha}}\left(\left(u_{\alpha}\right)_{c}\right)_{n}^{2} \\
& +\varepsilon^{2} F_{u^{\prime \prime} u^{\prime}}\left(u_{c}^{\prime \prime}\right)_{n}\left(u_{c}^{\prime}\right)_{n}+\varepsilon^{2} F_{u^{\prime \prime} u}\left(u_{c}^{\prime \prime}\right)_{n}\left(u_{c}\right)_{n} \\
& +\varepsilon^{2} F_{u^{\prime \prime} u_{\alpha}}\left(u_{c}^{\prime \prime}\right)_{n}\left(\left(u_{\alpha}\right)_{c}\right)_{n}+\varepsilon^{2} F_{u^{\prime} u}\left(u_{c}^{\prime}\right)_{n}\left(u_{c}\right)_{n} \\
& +\varepsilon^{2} F_{u^{\prime} u_{\alpha}}\left(u_{c}^{\prime}\right)_{n}\left(\left(u_{\alpha}\right)_{c}\right)_{n}+\varepsilon^{2} F_{u u_{\alpha}}\left(u_{c}\right)_{n}\left(\left(u_{\alpha}\right)_{c}\right)_{n} \\
= & -\varepsilon F_{\varepsilon}-F-\frac{1}{2} \varepsilon^{2} F_{\varepsilon \varepsilon} .
\end{aligned}
$$

Equation (9) is a variable coefficient pantograph equation of order two. We repeat the previously mentioned iteration procedure until the approximation is sufficient within a userdefined threshold.

\section{Application of the Method to the Pantograph-Type Delay Differential Equations}

In order to illustrate the accuracy and applicability of the presented method, the new perturbation-iteration algorithm is applied to six pantograph-type delay differential equations of different types. For comparison purposes, the solution intervals of problems are chosen generally the same as those in the references. Yet, the solution intervals are enlarged in illustrative examples 3 and 5. For a further wider interval, the method should be modified such that the Taylor series expansions are made around points other than zero.

3.1. Illustrative Example 1. Consider the first-order linear pantograph differential equation [25]:

$$
u^{\prime}(x)+u(x)-\frac{1}{10} u\left(\frac{x}{5}\right)=-\frac{1}{10} e^{-(1 / 5) x}, \quad 0 \leq x \leq 1
$$

with the initial condition

$$
u(0)=1
$$

for which the exact solution is $u(x)=e^{-x}$ [25].

Equation (10) can be written in the following form:

$$
u^{\prime}(x)+u(x)-\varepsilon u\left(\frac{x}{5}\right)+\frac{1}{10} e^{-(1 / 5) x}=0,
$$

where $\varepsilon=0.1$ is assumed to be the perturbation parameter. The nonzero terms of $(7)$ in PIA $(1,1)$ algorithm are

$$
\begin{aligned}
F_{u^{\prime}} & =1, \\
F_{u} & =1, \\
F_{\varepsilon} & =-u_{n}\left(\frac{x}{5}\right), \\
F & =u_{n}^{\prime}(x)+u_{n}(x)+\frac{1}{10} e^{-(1 / 5) x} .
\end{aligned}
$$

Upon substituting the terms of (13), (7) reduces to

$$
\begin{aligned}
& \left(u_{c}^{\prime}\right)_{n}(x)+\left(u_{c}\right)_{n}(x) \\
& \quad=u_{n}\left(\frac{x}{5}\right)-\frac{u_{n}^{\prime}(x)+u_{n}(x)+(1 / 10) e^{-(1 / 5) x}}{0.1} .
\end{aligned}
$$

When applying the iteration formula (4), we select an initially assumed function. Here, we start with the following trivial solution which satisfies the given initial condition:

$$
u_{0}=1 \text {. }
$$


TABLE 1: Comparison of the absolute errors of the PIA $(1,1)$ method with other numerical methods for example 1.

\begin{tabular}{|c|c|c|c|c|c|}
\hline \multirow[t]{2}{*}{$x$} & VIM [22] & Taylor method [23] & Continuous method [24] & $\begin{array}{c}\text { JRC method } \\
\alpha=\beta=0.5[25]\end{array}$ & $\operatorname{PIA}(1,1)$ \\
\hline & $n=4$ & & & $N=64$ & $n=4$ \\
\hline $2^{-1}$ & $2.40 e-15$ & $1.24 e-10$ & $3.33 e-11$ & $3.93 e-14$ & $1.43 e-15$ \\
\hline $2^{-2}$ & $7.91 e-17$ & $9.74 e-11$ & $4.13 e-11$ & $2.16 e-14$ & $7.91 e-17$ \\
\hline $2^{-3}$ & $2.53 e-18$ & $7.00 e-11$ & $4.62 e-11$ & $1.66 e-15$ & $2.53 e-18$ \\
\hline $2^{-4}$ & $8.03 e-20$ & $9.14 e-11$ & $4.89 e-11$ & $6.21 e-15$ & $8.03 e-20$ \\
\hline $2^{-5}$ & $2.52 e-21$ & $5.28 e-11$ & $5.05 e-11$ & $3.68 e-14$ & $2.52 e-21$ \\
\hline $2^{-6}$ & $7.00 e-23$ & $1.95 e-11$ & $4.74 e-11$ & $4.54 e-14$ & $7.92 e-23$ \\
\hline
\end{tabular}

Using (4) and (14), the approximate solutions at each step become

$$
\begin{aligned}
u_{1} & =\frac{1}{10}-\frac{1}{8} e^{-(1 / 5) x}+\frac{41}{40} e^{-x} \\
u_{2} & =\frac{1}{10^{2}}+\frac{1}{320} e^{-(1 / 5) x}-\frac{5}{384} e^{-(1 / 25) x}+\frac{9599}{9600} e^{-x}, \\
u_{3} & =\frac{1}{10^{3}}-\frac{1}{76800} e^{-(1 / 5) x}+\frac{1}{3072} e^{-(1 / 25) x} \\
& -\frac{125}{95232} e^{-(1 / 125) x}+\frac{11904001}{11904000} e^{-x}, \\
u_{4} & =\frac{1}{10^{4}}+\frac{1}{95232000} e^{-(1 / 5) x}-\frac{1}{737280} e^{-(1 / 25) x} \\
& +\frac{25}{761825} e^{-(1 / 125) x}-\frac{15625}{118849536} e^{-(1 / 625) x} \\
& +\frac{74280959999}{74280960000} e^{-x}
\end{aligned}
$$

Table 1 gives a comparison of the absolute errors of the PIA $(1,1)$ method with those of other numerical methods. It is apparent from the table that the absolute errors of $\operatorname{PIA}(1,1)$ method are smaller than those of the other methods for $n=4$, and also, considering the first four iteration solutions given in (16), one may notice that the numerical solution converges to the exact solution. Absolute errors obtained by PIA $(1,1)$ method are almost the same as those obtained by the Variational Iteration Method (VIM) [22] for $n=4$.

3.2. Illustrative Example 2. Consider the first-order linear pantograph differential equation [3]:

$$
u^{\prime}(x)+u(x)+u(0.8 x)=0, \quad 0 \leq x \leq 1
$$

with the initial condition

$$
u(0)=1
$$

which does not have an exact solution. Equation (17) can be written in the following form:

$$
u^{\prime}(x)+u(x)+\varepsilon u(0.8 x)=0,
$$

where $\varepsilon$ is the artificially introduced perturbation parameter. The nonzero terms of $(7)$ in $\operatorname{PIA}(1,1)$ algorithm are

$$
\begin{aligned}
F_{u^{\prime}} & =1, \\
F_{u} & =1, \\
F_{\varepsilon} & =u_{n}(0.8 x), \\
F & =u_{n}^{\prime}(x)+u_{n}(x) .
\end{aligned}
$$

For this specific example, (7) reduces to

$$
\left(u_{c}^{\prime}\right)_{n}(x)+\left(u_{c}\right)_{n}(x)=-u_{n}(0.8 x)-u_{n}^{\prime}(x)-u_{n}(x) .
$$

When applying the iteration formula (4), we start with the following trivial solution as the initial function, which satisfies the given initial conditions:

$$
u_{0}=1 \text {. }
$$

Using (4) and (21), the successive iteration results are

$$
\begin{aligned}
u_{1} & =-1+2 e^{-x}, \\
u_{2} & =1+10 e^{-x}-10 e^{-(4 / 5) x}, \\
u_{3} & =-1+\frac{218}{9} e^{-x}-50 e^{-(4 / 5) x}+\frac{250}{9} e^{-(16 / 25) x}, \\
u_{4} & =1+\frac{21490}{549} e^{-x}-\frac{1090}{9} e^{-(4 / 5) x}+\frac{1250}{9} e^{-(16 / 25) x} \\
& -\frac{31250}{549} e^{-(64 / 125) x}
\end{aligned}
$$

Table 2 shows the $\operatorname{PIA}(1,1)$ solutions of (17) for iteration numbers $n=5,6,7,8,9$. As stated above, we do not know the exact solution; therefore, we estimate a solution and observe a convergence.

A comparison of the results obtained by several different methods is shown in Table 3. The numerical results are consistent.

3.3. Illustrative Example 3. Consider the first-order nonlinear pantograph differential equation [29]:

$$
u^{\prime}(x)=-2 u^{2}\left(\frac{x}{2}\right)+1, \quad 0 \leq x \leq 1
$$


TABLE 2: Convergence of the PIA $(1,1)$ solution for example 2.

\begin{tabular}{lccccc}
\hline$x_{i}$ & $u_{5}(x)$ & $u_{6}(x)$ & $u_{7}(x)$ & $u_{8}(x)$ & $u_{9}(x)$ \\
\hline 0 & 1 & 1 & 1 & 1 & 1 \\
0.2 & 0.6646909 & 0.6646910 & 0.6646910 & 0.6646910 & 0.6646910 \\
0.4 & 0.4335604 & 0.4335607 & 0.4335607 & 0.4335607 & 0.4335607 \\
0.6 & 0.2764790 & 0.2764824 & 0.2764823 & 0.2764823 & 0.2764823 \\
0.8 & 0.1714677 & 0.1714846 & 0.1714841 & 0.1714841 & 0.1714841 \\
1 & 0.1026142 & 0.1026723 & 0.1026700 & 0.1026701 & 0.1026701 \\
\hline
\end{tabular}

with the initial condition

$$
u(0)=0
$$

which has the exact solution $u(x)=\sin (x)$.

Equation (24) can be written in the following form:

$$
u^{\prime}(x)+\varepsilon u^{2}\left(\frac{x}{2}\right)-1=0
$$

where $\varepsilon=2$ is assumed to be the perturbation parameter. The nonzero terms of $(7)$ in $\operatorname{PIA}(1,1)$ algorithm are

$$
\begin{aligned}
F_{u^{\prime}} & =1, \\
F_{\varepsilon} & =u_{n}^{2}\left(\frac{x}{2}\right), \\
F & =u_{n}^{\prime}(x)-1 .
\end{aligned}
$$

The $n$th correction term of the perturbation expansion in (4) is

$$
\left(u_{c}^{\prime}\right)_{n}(x)=-u_{n}^{2}\left(\frac{x}{2}\right)-\frac{u_{n}^{\prime}(x)-1}{2} .
$$

In this example, we start with the initial guess:

$$
u_{0}=x
$$

Thus, the successive approximations are

$$
\begin{aligned}
u_{1}= & x-\frac{1}{6} x^{3} \\
u_{2}= & x-\frac{1}{6} x^{3}+\frac{1}{120} x^{5}-\frac{1}{8064} x^{7} \\
u_{3}= & x-\frac{1}{6} x^{3}+\frac{1}{120} x^{5}-\frac{1}{5040} x^{7}+\frac{61}{23224320} x^{9} \\
& -\frac{67}{3406233600} x^{11}+\frac{1}{12881756160} x^{13} \\
& -\frac{1}{7990652436480} x^{15}
\end{aligned}
$$

Subsequently, we determine the absolute maximum error for $u_{n}$ as

$$
\begin{aligned}
E_{n} & =\left\|u_{n}(x)-u(x)\right\|_{\infty} \\
& =\max \left\{\left|u_{n}(x)-u(x)\right|, 0 \leq x \leq 1\right\}
\end{aligned}
$$

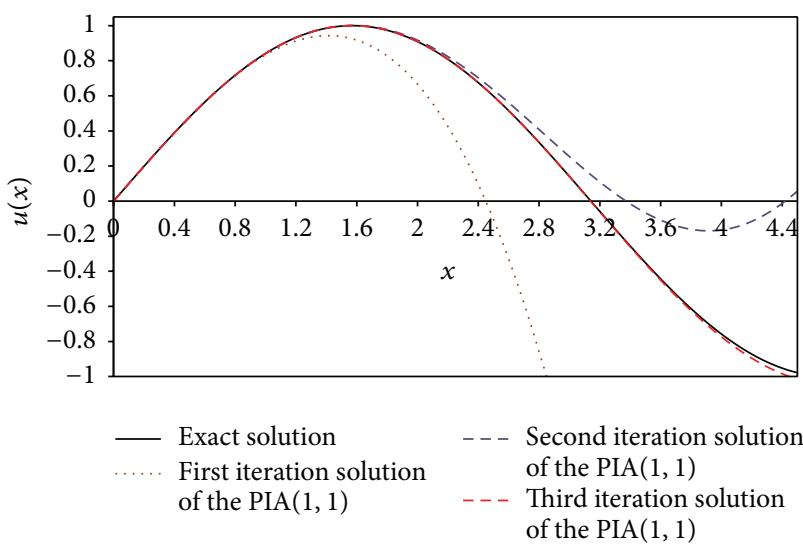

Figure 1: Comparison of the first three iteration solutions of the PIA $(1,1)$ method with the exact solution for example 3 .

The absolute maximum errors $E_{n}$ for different values of $n$ are given in Table 4 . Note that the error decreases continually as $n$ increases.

The same problem is solved in various studies and it is seen that the absolute maximum error for solving this example via Homotopy Asymptotic Method is $1.2 \times 10^{-6}$ [30] and via Optimal Homotopy Asymptotic Method is $4 \times$ $10^{-8}$ [29] while the absolute maximum error obtained by the $\operatorname{PIA}(1,1)$ method is $5.35 \times 10^{-20}$. This affirmed that the presented method is more accurate than the other two methods.

In Figure 1, the first three iteration solutions ( $n=1,2,3)$ of the PIA $(1,1)$ method contrasted with the exact solution. It is revealed that even when the solution interval of the problem is expanded, $\operatorname{PIA}(1,1)$ method goes on to yield convergent numerical solutions.

3.4. Illustrative Example 4. Consider the initial value problem of second-order differential equation with pantograph delay [31]:

$$
\begin{array}{r}
u^{\prime \prime}(x)=-u\left(\frac{x}{2}\right)-u^{2}(x)+\sin ^{4}(x)+\sin ^{2}(x)+8 \\
x \geq 0
\end{array}
$$

with initial conditions

$$
\begin{gathered}
u(0)=2, \\
u^{\prime}(0)=0
\end{gathered}
$$

which has the exact solution $u(x)=(5-\cos 2 x) / 2$.

3.4.1. PIA(1,1) Solution. Equation (32) can be written in the following form:

$$
\begin{gathered}
u^{\prime \prime}(x)+\varepsilon\left(u\left(\frac{x}{2}\right)+u^{2}(x)\right) \\
=\sin ^{4}(x)+\sin ^{2}(x)+8
\end{gathered}
$$


TABLE 3: Comparison of the PIA $(1,1)$ solution with other numerical methods for example 2.

\begin{tabular}{|c|c|c|c|c|c|c|}
\hline \multirow[t]{2}{*}{$x_{i}$} & \multirow{2}{*}{ Walsh series method [26] } & \multirow{2}{*}{$\begin{array}{c}\text { DUSF series method } \\
\text { [27] } \\
m=100 \\
h=0.01 \\
\end{array}$} & \multirow{2}{*}{$\begin{array}{c}\text { Hermite series } \\
\text { method [3] } \\
\quad N=8\end{array}$} & \multirow{2}{*}{$\begin{array}{l}\text { Taylor series } \\
\text { method [4] } \\
\qquad N=8\end{array}$} & \multirow{2}{*}{$\begin{array}{l}\text { Laguerre matrix } \\
\text { method [28] } \\
\qquad N=8\end{array}$} & \multirow{2}{*}{$\begin{array}{c}\operatorname{PIA}(1,1) \\
n=8\end{array}$} \\
\hline & & & & & & \\
\hline 0 & 1 & 1 & 1 & 1 & 1 & 1 \\
\hline 0.2 & 0.665621 & 0.664677 & 0.664691 & 0.664691 & 0.6646910 & 0.6646910 \\
\hline 0.4 & 0.432426 & 0.433540 & 0.433561 & 0.433561 & 0.4335607 & 0.4335607 \\
\hline 0.6 & 0.275140 & 0.276460 & 0.276482 & 0.276482 & 0.2764831 & 0.2764823 \\
\hline 0.8 & 0.170320 & 0.171464 & 0.171484 & 0.171484 & 0.1714942 & 0.1714841 \\
\hline 1 & 0.100856 & 0.102652 & 0.102670 & 0.102744 & 0.1027437 & 0.1026701 \\
\hline
\end{tabular}

TABLE 4: The absolute maximum errors $E_{n}$ for example 3.

\begin{tabular}{ccccccc}
\hline$n$ & 1 & 2 & 3 & 4 & 5 & 6 \\
\hline$E_{n}$ & $8.13 e-3$ & $7.16 e-5$ & $1.23 e-7$ & $4.38 e-11$ & $2.91 e-15$ & $5.35 e-20$ \\
\hline
\end{tabular}

where $\varepsilon=1$ is the artificially introduced perturbation parameter. Using (3), (34) returns to

$$
\begin{aligned}
F\left(u^{\prime \prime}, u^{\prime}, u, u_{\alpha}, \varepsilon\right)= & u^{\prime \prime}(x)+\varepsilon\left(u\left(\frac{x}{2}\right)+u^{2}(x)\right) \\
& -\left(\sin ^{4}(x)+\sin ^{2}(x)+8\right)=0 .
\end{aligned}
$$

The nonzero terms of $(7)$ in $\operatorname{PIA}(1,1)$ algorithm are

$$
\begin{aligned}
F_{u^{\prime \prime}} & =1, \\
F_{\varepsilon} & =u_{n}\left(\frac{x}{2}\right)+u_{n}^{2}(x), \\
F & =u_{n}^{\prime \prime}(x)-\sin ^{4}(x)-\sin ^{2}(x)-8 .
\end{aligned}
$$

Substituting (36), (32) reduces to

$$
\begin{aligned}
\left(u_{c}^{\prime \prime}\right)_{n}(x)= & -u_{n}\left(\frac{x}{2}\right)-u_{n}^{\prime \prime}(x)-u_{n}^{2}(x)+\sin ^{4}(x) \\
& +\sin ^{2}(x)+8
\end{aligned}
$$

The initial trial function is selected as

$$
u_{0}=2 \text {, }
$$

and using (37), the approximate first iteration solution is obtained as

$$
\begin{aligned}
u_{1} & =\frac{13}{16}-\frac{1}{16} \sin ^{4}(x)+\frac{3}{16} \cos ^{2}(x)+\frac{23 x^{2}}{16} \\
& +\cos ^{2}\left(\frac{x}{2}\right)
\end{aligned}
$$

Even the first iteration of $\operatorname{PIA}(1,1)$ solution, $u_{1}$, gave the same result as the first iteration solution obtained by the VIM [31]. Still, the second and third iteration solutions of the problem are better than those of the VIM solutions as observed in Figures 2 and 3.

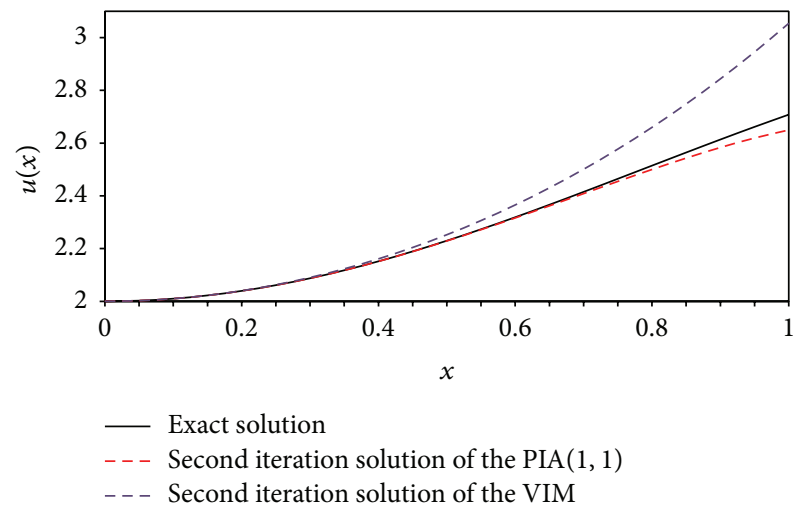

FIGURE 2: Comparison of the second iteration solutions of the VIM and PIA $(1,1)$ with exact solution for example 4 .

3.4.2. $\operatorname{PIA}(1,2)$ Solution. In order to apply the $\operatorname{PIA}(1,2)$ algorithm obtained by (9), we have to write (32) in the following form:

$$
\begin{aligned}
F\left(u^{\prime \prime}, u^{\prime}, u, u_{\alpha}, \varepsilon\right)= & u^{\prime \prime}(x)+\varepsilon u^{2}(x)+\varepsilon^{2} u\left(\frac{x}{2}\right) \\
& -\left(\sin ^{4}(x)+\sin ^{2}(x)+8\right)=0
\end{aligned}
$$

where $\varepsilon$ is the artificially introduced perturbation parameter. The nonzero terms of $(9)$ in $\operatorname{PIA}(1,2)$ algorithm are

$$
\begin{aligned}
F_{u^{\prime \prime}} & =1, \\
F_{u \varepsilon} & =2 u_{n}(x), \\
F_{\varepsilon} & =u_{n}^{2}(x), \\
F_{\varepsilon \varepsilon} & =2 u_{n}\left(\frac{x}{2}\right), \\
F & =u_{n}^{\prime \prime}(x)-\sin ^{4}(x)-\sin ^{2}(x)-8 .
\end{aligned}
$$




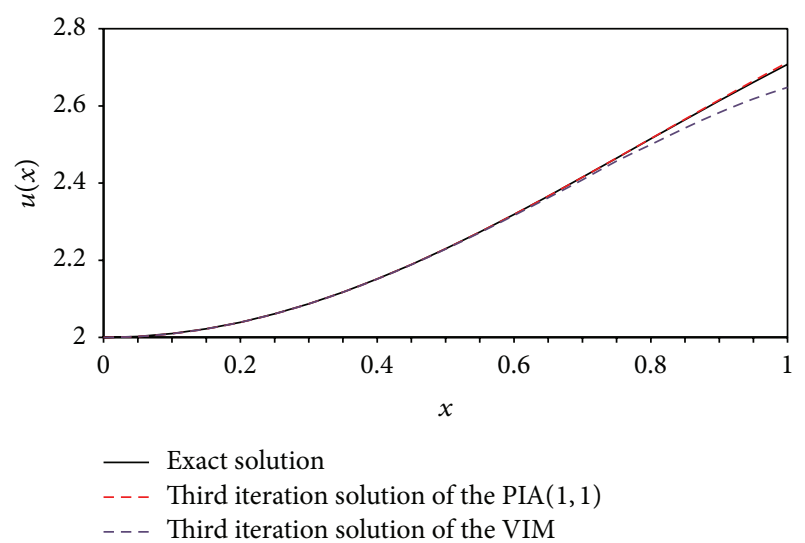

FIGURE 3: The comparison of the third iteration solutions of the VIM and PIA $(1,1)$ with exact solution for example 4.

Note that introducing the small parameter $\varepsilon=1$ as a coefficient of the pantograph term simplifies (40) and makes it easily solvable. For this specific example, (40) reads

$$
\begin{aligned}
& \left(u_{c}^{\prime \prime}\right)_{n}(x)+\left(2 u_{n}(x)\right)\left(u_{c}\right)_{n}(x) \\
& =-u_{n}\left(\frac{x}{2}\right)-u_{n}^{\prime \prime}(x)-u_{n}^{2}(x)+\sin ^{4}(x)+\sin ^{2}(x) \\
& \quad+8
\end{aligned}
$$

When applying the iteration formula (4), we select the following initially assumed function:

$$
u_{0}=2 \text {, }
$$

and using (9), the approximate solution is

$$
\begin{aligned}
u_{1}= & \frac{263}{96}-\frac{49}{96} \cos (2 x)-\frac{1}{12} \cos ^{4}(2 x)+\frac{1}{48} \cos ^{2}(x) \\
& -\frac{1}{4} x \sin (x) \cos (x)-\frac{1}{6} \cos (x) .
\end{aligned}
$$

First iteration of PIA $(1,2)$ and second and third iterations of VIM solutions are compared with the exact solution. As can be seen from Figure 4, even the first iteration of PIA $(1,2)$ yields much better results than the first three iteration solutions of the VIM.

3.5. Illustrative Example 5. Consider the initial value problem of nonlinear variable coefficient differential equation with pantograph delay [6]:

$$
u^{\prime \prime}(x)=u(x)-\frac{8}{x^{2}} u^{2}\left(\frac{x}{2}\right), \quad x \geq 0
$$

with initial conditions

$$
\begin{gathered}
u(0)=0, \\
u^{\prime}(0)=1
\end{gathered}
$$

which has the exact solution $u(x)=x e^{-x}$.

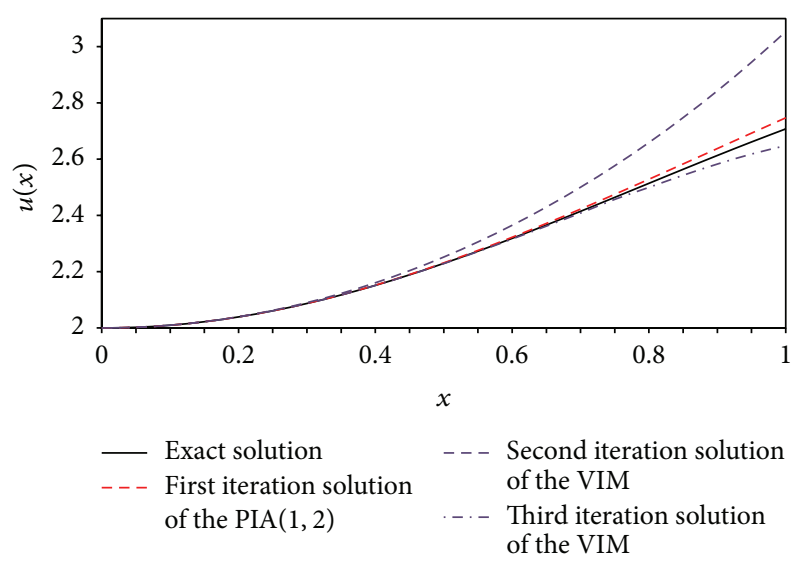

FIgURE 4: The comparison of second and third iteration solutions of the VIM and first iteration solution of the PIA $(1,2)$ with exact solution of example 4 .

Equation (45) can be written in the following form:

$$
u^{\prime \prime}(x)+\varepsilon\left(\frac{8}{x^{2}} u^{2}\left(\frac{x}{2}\right)-u(x)\right)=0,
$$

where $\varepsilon=1$ is the artificially introduced perturbation parameter. Using (3), (45) returns to

$$
\begin{aligned}
F\left(u^{\prime \prime}, u^{\prime}, u, u_{\alpha}, \varepsilon\right) & =u^{\prime \prime}(x)+\varepsilon\left(\frac{8}{x^{2}} u^{2}\left(\frac{x}{2}\right)-u(x)\right) \\
& =0 .
\end{aligned}
$$

The nonzero terms in $\operatorname{PIA}(1,1)$ algorithm are

$$
\begin{aligned}
F_{u^{\prime \prime}} & =1, \\
F_{\varepsilon} & =\frac{8}{x^{2}} u_{n}^{2}\left(\frac{x}{2}\right)-u_{n}(x), \\
F & =u_{n}^{\prime \prime}(x) .
\end{aligned}
$$

Substituting (49), (45) reduces to

$$
\left(u_{c}^{\prime \prime}\right)_{n}(x)=-\frac{8}{x^{2}} u_{n}^{2}\left(\frac{x}{2}\right)+u_{n}(x)-u_{n}^{\prime \prime}(x) .
$$

The initial trial function is selected as

$$
u_{0}(x)=x,
$$

and using (50), the approximate first iteration solution is obtained as

$$
\begin{gathered}
u_{1}=x-x^{2}+\frac{1}{6} x^{3} \\
u_{2}=x-x^{2}+\frac{1}{2} x^{3}-\frac{5}{36} x^{4}+\frac{1}{80} x^{5}-\frac{1}{8640} x^{6}
\end{gathered}
$$

The absolute maximum errors $E_{n}$ for different values of $n$ are given in Table 5 , and it is revealed that the error decreases continually as $n$ increases. 
TABLE 5: The absolute maximum errors $E_{n}$ for example 5.

\begin{tabular}{cccccccc}
\hline$n$ & 1 & 2 & 3 & 4 & 5 & 6 & 7 \\
\hline$E_{n}$ & $2.01 e-1$ & $5.61 e-3$ & $1.59 e-5$ & $2.75 e-7$ & $3.68 e-9$ & $6.76 e-11$ & $4.94 e-13$ \\
\hline
\end{tabular}

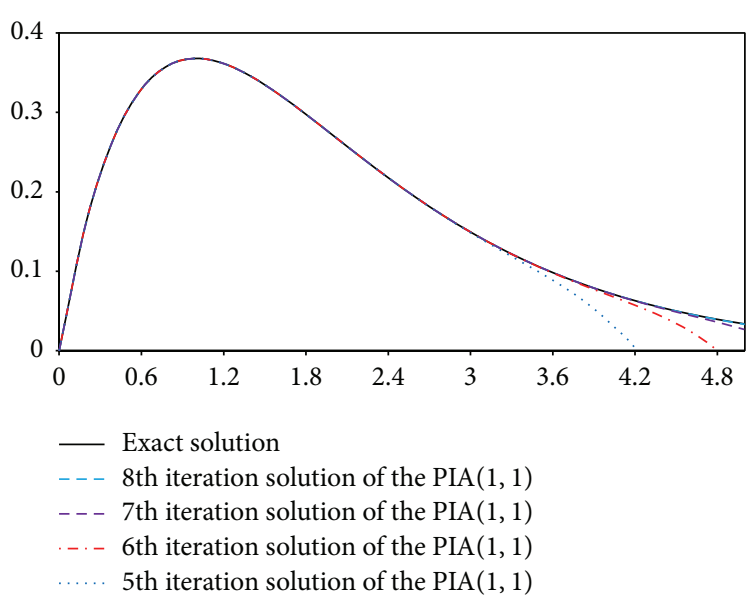

Figure 5: Comparison of the iteration solutions of the $\operatorname{PIA}(1,1)$ method with the exact solution for example 5 .

Figure 5 illustrates the $\operatorname{PIA}(1,1)$ solution of this example in the interval $[0,5]$. In this example, we enlarge the interval so as to compare our solution more thoroughly with the exact solution. The present method yields pretty good results.

3.6. Illustrative Example 6. Consider the second-order nonlinear pantograph functional differential equation $[7,8]$ :

$$
u^{\prime \prime}(x)=\left([u(x)]^{2}+|u(x)|^{3}\right) u\left(\frac{x}{2}\right), \quad x \geq 0
$$

with initial conditions

$$
u(0)=1, \quad u(1)=\frac{1}{2}
$$

which has the exact solution $u(x)=1 /(1+x)$.

Equation (45) can be written in the following form:

$$
u^{\prime \prime}(x)-\varepsilon\left(\left([u(x)]^{2}+|u(x)|^{3}\right) u\left(\frac{x}{2}\right)\right)=0,
$$

where $\varepsilon$ is the artificially introduced small parameter. Using (3), (45) returns to

$$
\begin{aligned}
F & \left(u^{\prime \prime}, u^{\prime}, u, u_{\alpha}, \varepsilon\right) \\
& =u^{\prime \prime}(x)-\varepsilon\left(\left([u(x)]^{2}+|u(x)|^{3}\right) u\left(\frac{x}{2}\right)\right)=0 .
\end{aligned}
$$

The nonzero terms in $\operatorname{PIA}(1,1)$ algorithm are

$$
\begin{aligned}
F_{u^{\prime \prime}} & =1, \\
F_{\varepsilon} & =-\left(\left([u(x)]^{2}+\left|u_{n}(x)\right|^{3}\right) u_{n}\left(\frac{x}{2}\right)\right), \\
F & =u_{n}^{\prime \prime}(x) .
\end{aligned}
$$

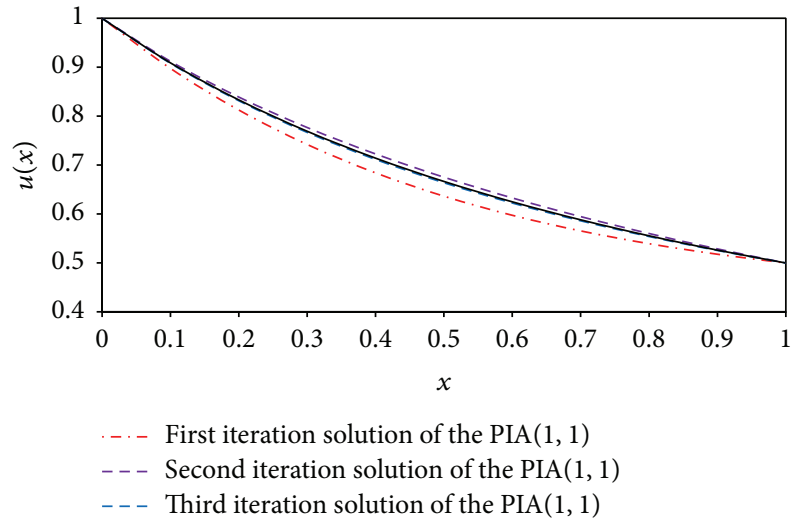

Figure 6: Comparison of the first three iteration solutions of the PIA $(1,1)$ method with the exact solution for example 6 .

Substituting (49), (45) reduces to

$$
\begin{aligned}
\left(u_{c}^{\prime \prime}\right)_{n}(x)= & -u_{n}^{\prime \prime}(x) \\
& +\left(\left([u(x)]^{2}+\left|u_{n}(x)\right|^{3}\right) u_{n}\left(\frac{x}{2}\right)\right) .
\end{aligned}
$$

The initial trial function is selected as

$$
u_{0}(x)=1-\frac{x}{2}
$$

and using (50), the approximate first iteration solution is obtained as

$$
\begin{aligned}
& u_{1}(x)=1-\frac{1073}{960} x+x^{2}-\frac{1}{2} x^{3}+\frac{13}{96} x^{4}-\frac{3}{160} x^{5} \\
& +\frac{1}{960} x^{6}
\end{aligned}
$$

In Figure 6, first three iteration solutions obtained by the PIA $(1,1)$ algorithm are compared with the exact solution. The convergence of iteration solutions to the exact solution is obvious.

Figure 7 illustrates the absolute error functions of the first three iteration solutions of the $\operatorname{PIA}(1,1)$ method. The maximum absolute error decreases from 0,03013 in the first iteration to 0,00262 in the third iteration.

\section{Conclusion}

The new perturbation-iteration method is employed for the first time to numerically solve the pantograph equations. The comparative results showed that the method is highly 


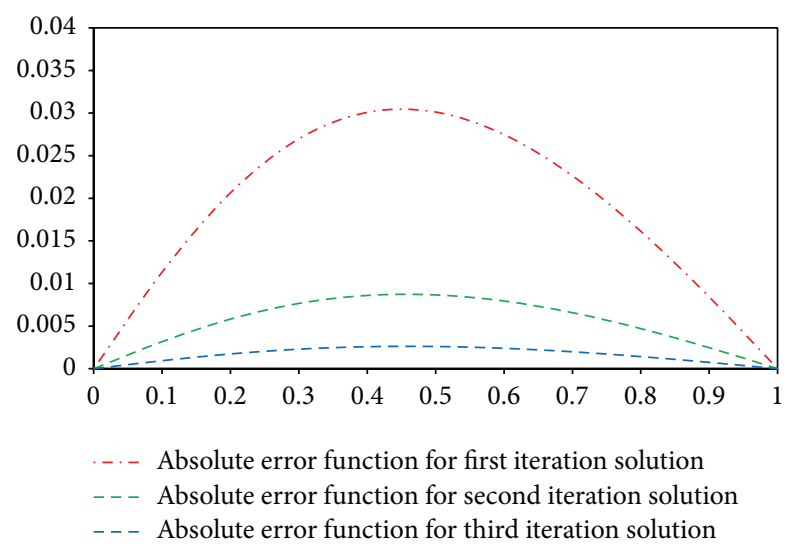

FIGURE 7: Comparison of the absolute error functions of the first three iteration solutions of the $\operatorname{PIA}(1,1)$ method with the exact solution for example 6 .

efficient and reliable in both linear and nonlinear problems. While, for the linear problems studied, PIA $(1,1)$ algorithm gave almost the same results as Variational Iteration Method, it gave better results for the nonlinear problems. On the other hand, the results obtained in the first iteration of the PIA $(1,2)$ algorithm are more convergent than those obtained in the third iteration of the Variational Iteration Method; this validates the performance of the present method. Although the equations of $\operatorname{PIA}(1,2)$ algorithm are more entangled than those of PIA $(1,1)$, faster convergence is achieved with PIA $(1,2)$ when compared to $\operatorname{PIA}(1,1)$.

It is conceived that the present method can be useful in developing new algorithms since they can be generated in various forms according to the number of correction terms in perturbation expansion and the order of derivation in Taylor expansion.

The disadvantage of the method is that the number of terms in the solution function increases seriously.

In this study, the solution intervals of problems are chosen generally the same as those in the references, for comparison purposes. In three of the examples, the solution intervals are extended moderately; yet, for a wider solution interval, the method should be modified such that the Taylor series expansions are made around points other than zero. The method can also be extended to other types of delay differential equations but some modifications are required.

\section{Conflict of Interests}

The authors declare that there is no conflict of interests regarding the publication of this paper.

\section{References}

[1] M.Z.Liu and D. Li, "Properties of analytic solution and numerical solution of multi-pantograph equation," Applied Mathematics and Computation, vol. 155, no. 3, pp. 853-871, 2004.

[2] Y. Muroya, E. Ishiwata, and H. Brunner, "On the attainable order of collocation methods for pantograph integrodifferential equations," Journal of Computational and Applied Mathematics, vol. 152, no. 1-2, pp. 347-366, 2003.
[3] S. Yalçınbaş, M. Aynigül, and M. Sezer, "A collocation method using Hermite polynomials for approximate solution of pantograph equations," Journal of the Franklin Institute, vol. 348, no. 6, pp. 1128-1139, 2011.

[4] M. Sezer and A. Akyüz-Daşcıoğlu, "A Taylor method for numerical solution of generalized pantograph equations with linear functional argument," Journal of Computational and Applied Mathematics, vol. 200, no. 1, pp. 217-225, 2007.

[5] S. Sedaghat, Y. Ordokhani, and M. Dehghan, "Numerical solution of the delay differential equations of pantograph type via Chebyshev polynomials," Communications in Nonlinear Science and Numerical Simulation, vol. 17, no. 12, pp. 4815-4830, 2012.

[6] B. Benhammouda, H. Vazquez-Leal, and L. HernandezMartinez, "Procedure for exact solutions of nonlinear pantograph delay differential equations," British Journal of Mathematics \& Computer Science, vol. 4, no. 19, pp. 2738-2751, 2014.

[7] S. Abbasbandy and C. Bervillier, "Analytic continuation of Taylor series and the boundary value problems of some nonlinear ordinary differential equations," Applied Mathematics and Computation, vol. 218, no. 5, pp. 2178-2199, 2011.

[8] M. A. Z. Raja, "Numerical treatment for boundary value problems of Pantograph functional differential equation using computational intelligence algorithms," Applied Soft Computing, vol. 24, pp. 806-821, 2014.

[9] D. Trif, "Direct operatorial tau method for pantograph-type equations," Applied Mathematics and Computation, vol. 219, no. 4, pp. 2194-2203, 2012.

[10] A. H. Nayfeh, Introduction to Perturbation Techniques, John Wiley \& Sons, New York, NY, USA, 1981.

[11] J.-H. He, "Iteration perturbation method for strongly nonlinear oscillations," Journal of Vibration and Control, vol. 7, no. 5, pp. 631-642, 2001.

[12] R. E. Mickens, "Iteration method solutions for conservative and limit-cycle $\mathrm{x}^{1 / 3}$ force oscillators," Journal of Sound and Vibration, vol. 292, no. 3-5, pp. 964-968, 2006.

[13] Y. Aksoy, M. Pakdemirli, S. Abbasbandy, and H. Boyacı, "New perturbation-iteration solutions for nonlinear heat transfer equations," International Journal of Numerical Methods for Heat \& Fluid Flow, vol. 22, no. 7, pp. 814-828, 2012.

[14] T. Öziş and A. Yıldırım, "Generating the periodic solutions for forcing van der Pol oscillators by the iteration perturbation method," Nonlinear Analysis. Real World Applications, vol. 10, no. 4, pp. 1984-1989, 2009.

[15] D. D. Ganji, S. Karimpour, and S. S. Ganji, "He’s iteration perturbation method to nonlinear oscillations of mechanical systems with single-degree-of freedom," International Journal of Modern Physics B, vol. 23, no. 11, pp. 2469-2477, 2009.

[16] V. Marinca and N. Herisanu, "A modified iteration perturbation method for some nonlinear oscillation problems," Acta Mechanica, vol. 184, no. 1-4, pp. 231-242, 2006.

[17] M. Pakdemirli and H. Boyac1, "Generation of root finding algorithms via perturbation theory and some formulas," Applied Mathematics and Computation, vol. 184, no. 2, pp. 783-788, 2007.

[18] M. Pakdemirli, H. Boyacı, and H. A. Yurtsever, "Perturbative derivation and comparisons of root-finding algorithms with fourth order derivatives," Mathematical \& Computational Applications, vol. 12, no. 2, pp. 117-124, 2007.

[19] M. Pakdemirli, H. Boyacı, and H. A. Yurtsever, "A root finding algorithm with fifth order derivatives," Mathematical \& Computational Applications, vol. 13, no. 2, pp. 123-128, 2008. 
[20] Y. Aksoy and M. Pakdemirli, "New perturbation-iteration solutions for Bratu-type equations," Computers \& Mathematics with Applications, vol. 59, no. 8, pp. 2802-2808, 2010.

[21] İ. T. Dolapçı, M. Şenol, and M. Pakdemirli, "New perturbation iteration solutions for Fredholm and Volterra integral equations," Journal of Applied Mathematics, vol. 2013, Article ID 682537, 5 pages, 2013.

[22] A. Saadatmandi and M. Dehghan, "Variational iteration method for solving a generalized pantograph equation," Computers \& Mathematics with Applications, vol. 58, no. 11-12, pp. 2190-2196, 2009.

[23] M. Sezer, S. Yalçınbaş, and M. Gülsu, "A Taylor polynomial approach for solving generalized pantograph equations with nonhomogenous term," International Journal of Computer Mathematics, vol. 85, no. 7, pp. 1055-1063, 2008.

[24] X. Y. Li and B. Y. Wu, "A continuous method for nonlocal functional differential equations with delayed or advanced arguments," Journal of Mathematical Analysis and Applications, vol. 409, no. 1, pp. 485-493, 2014.

[25] E. H. Doha, A. H. Bhrawy, D. Baleanu, and R. M. Hafez, "A new Jacobi rational-Gauss collocation method for numerical solution of generalized pantograph equations," Applied Numerical Mathematics, vol. 77, pp. 43-54, 2014.

[26] G. P. Rao and K. R. Palanisamy, "Walsh stretch matrices and functional-differential equations," IEEE Transactions on Automatic Control, vol. 27, no. 1, pp. 272-276, 1982.

[27] C. Hwang, "Solution of a functional differential equation via delayed unit step functions," International Journal of Systems Science, vol. 14, no. 9, pp. 1065-1073, 1983.

[28] Ş. Yüzbaşı, E. Gök, and M. Sezer, "Laguerre matrix method with the residual error estimation for solutions of a class of delay differential equations," Mathematical Methods in the Applied Sciences, vol. 37, no. 4, pp. 453-463, 2014.

[29] N. R. Anakira, A. K. Alomari, and I. Hashim, "Optimal homotopy asymptotic method for solving delay differential equations," Mathematical Problems in Engineering, vol. 2013, Article ID 498902, 11 pages, 2013.

[30] A. K. Alomari, M. S. Noorani, and R. Nazar, "Solution of delay differential equation by means of homotopy analysis method," Acta Applicandae Mathematicae, vol. 108, no. 2, pp. 395-412, 2009.

[31] H. Liu, A. Xiao, and L. Su, "Convergence of variational iteration method for second-order delay differential equations," Journal of Applied Mathematics, vol. 2013, Article ID 634670, 9 pages, 2013. 


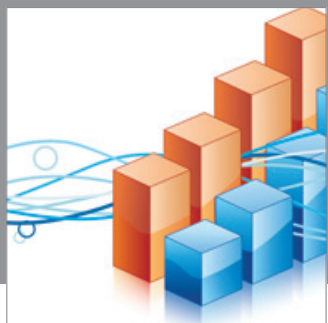

Advances in

Operations Research

mansans

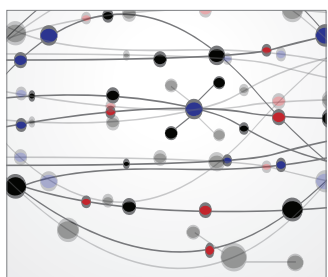

The Scientific World Journal
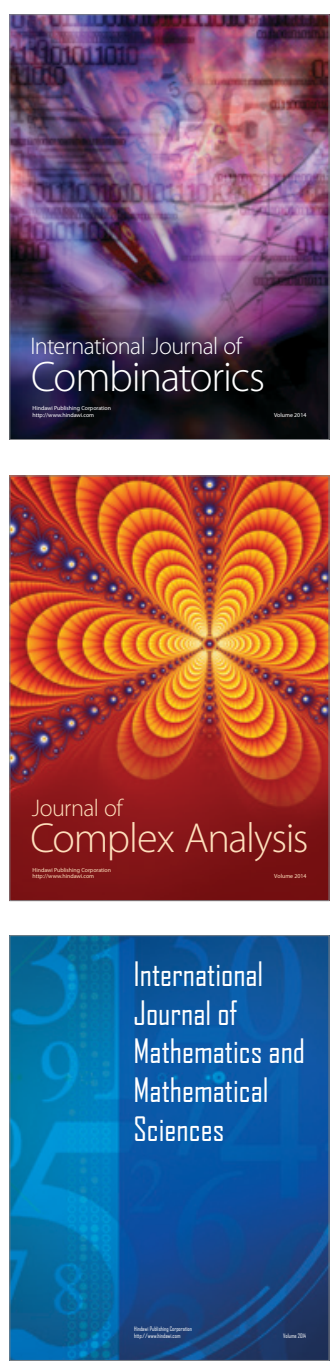
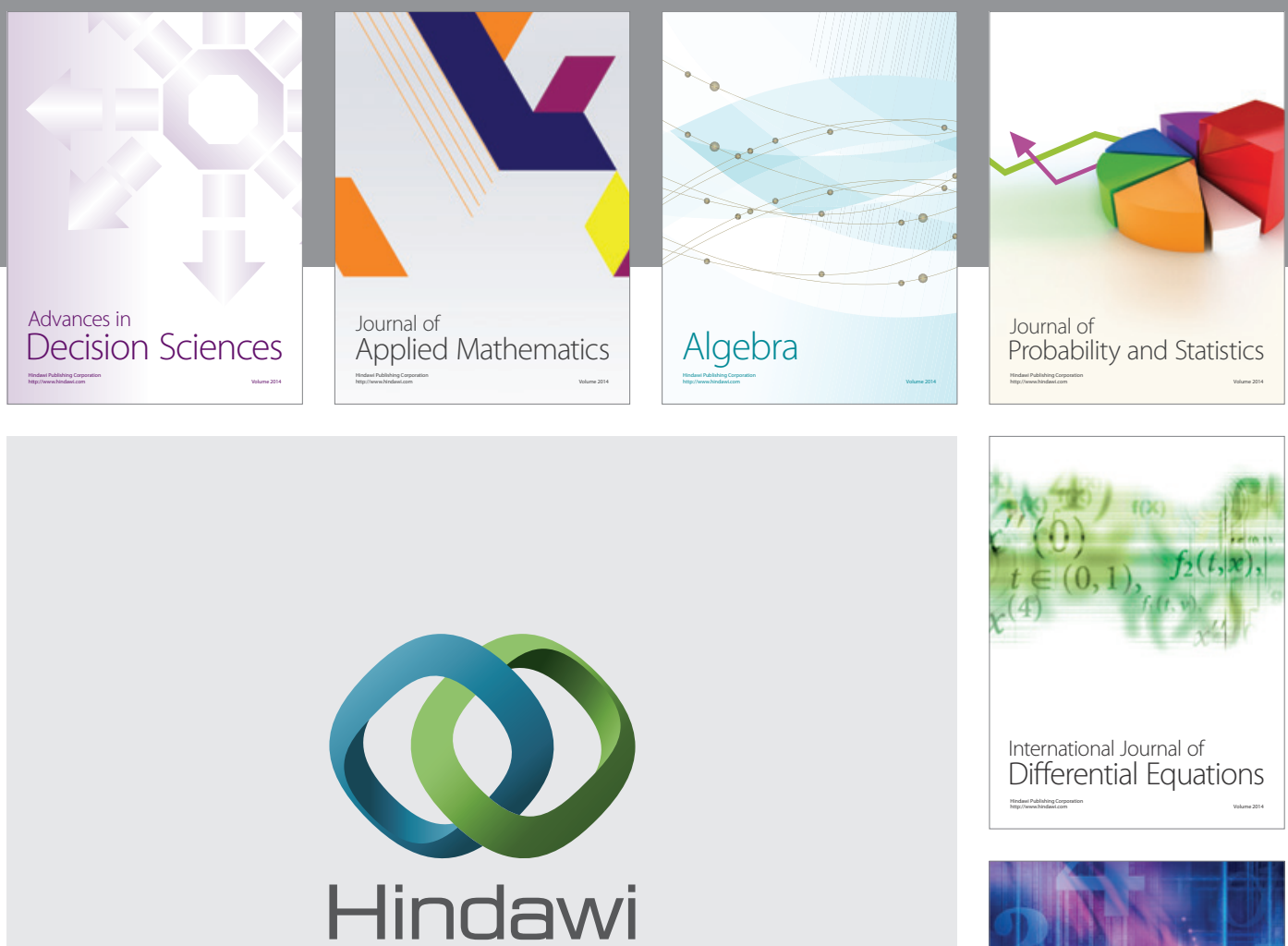

Submit your manuscripts at http://www.hindawi.com
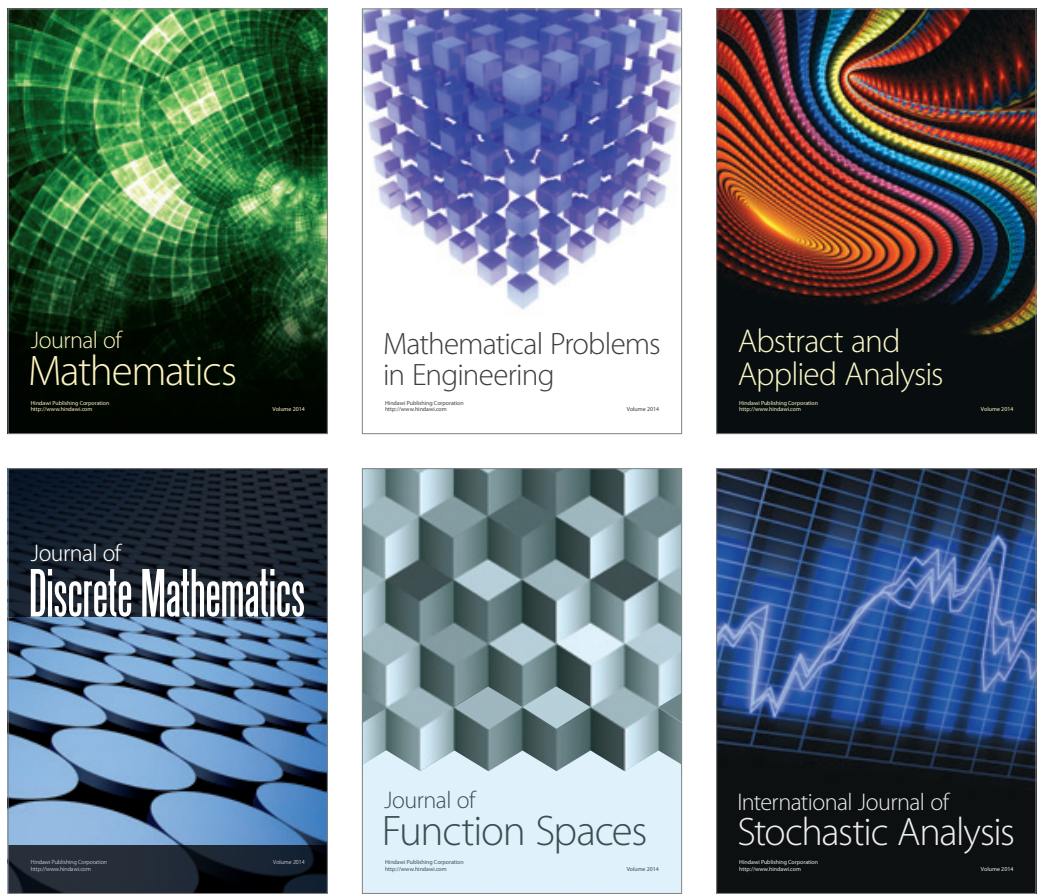

Journal of

Function Spaces

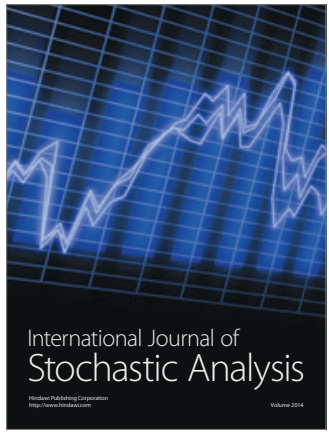

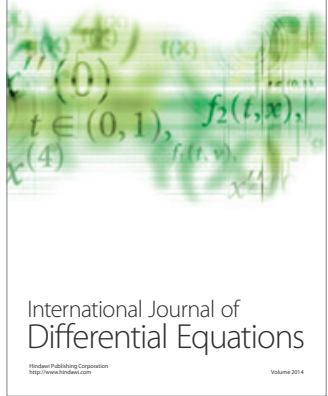
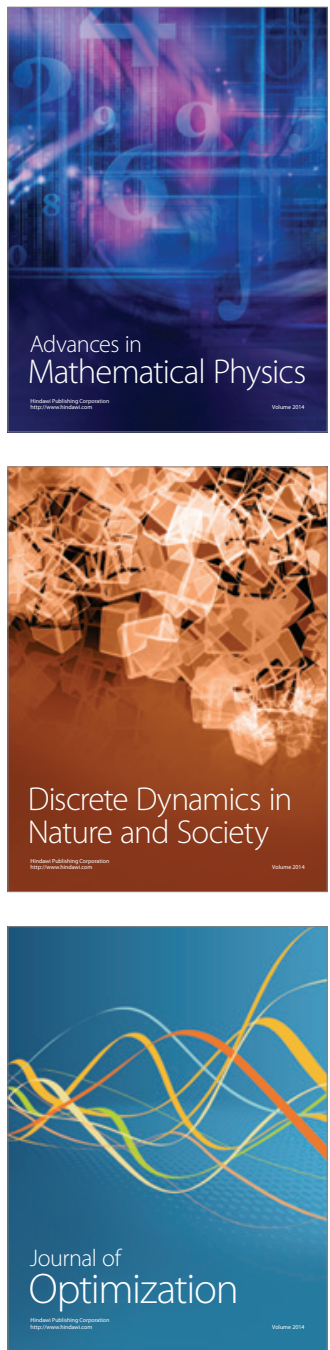